\title{
Campanha Maio Verde: avaliação do conhecimento populacional sobre o glaucoma
}

\author{
Maio Verde Campaign: evaluation of population awareness of glaucoma \\ Caroline Lopes Aragão de Macedo ${ }^{1}$ (D), Amanda Galdino Carneiro' ${ }^{1}$, Lucas Almeida Linhares ${ }^{1}$ (D), \\ Carlos Rodrigo Damasceno Feitosa de Santana' (1), João Augusto Lima Bisneto ${ }^{1}$ (D), José de Paula Barbosa Neto ${ }^{1}$ (D), \\ Matheus Carvalho Vasconcelos ${ }^{1}$ (i), Luis Eduardo Reis Amaral' ${ }^{1}$, Karine Estéfane Gonçalves ${ }^{1}$ (i), Anderson Costa Maia ${ }^{1}$ (i) \\ Hissa Tavares de Lima Gradvohl² (B), Juliana de Lucena Martins Ferreira ${ }^{3}$ [] \\ Centro Universitário Unichristus, Fortaleza, CE, Brasil. \\ ${ }^{2}$ Sociedade Cearense de Oftalmologia, Fortaleza, CE, Brasil. \\ ${ }^{3}$ Disciplina de Oftalmologia, Centro Universitário Unichristus, Fortaleza, CE, Brasil.
}

Macedo CL, Carneiro AG, Linhares LA, Santana CR, Lima Bisneto JA, Barbosa Neto JP, et al. Campanha Maio Verde: avaliação do conhecimento populacional sobre o glaucoma. Rev Bras Ofltalmol. 2021;80(5):e0031.

Descritores:

Glaucoma; Educação em saúde; Prevenção primária; Cegueira

Keywords:

Glaucoma; Health education; Primary prevention; Blindness

Submetido: $17 / 11 / 2020$

Aceito: 3/6/2021

Autor correspondente: Caroline Lopes Aragão de Macedo R. João Adolfo Gurgel 133 - Cocó, Fortaleza - CE, 60190-180 Tel.: (85) 99678-8638 E-mail: clopesaragao@gmail.com

Instituição de realização do trabalho: Centro Universitário Unichristus, Fortaleza, CE, Brasil

Fonte de auxílio à pesquisa: trabalho não financiado.

Conflitos de interesse: os autores declaram que não há conflitos de interesses.

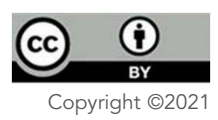

\section{RESUMO}

Objetivo: Avaliar o conhecimento da população sobre o glaucoma.

Métodos: Foi realizado um estudo transversal, descritivo, não controlado, cujo instrumento de coleta de dados foi um questionário contendo perguntas sobre o perfil sociodemográfico e o glaucoma, aplicado em indivíduos, antes e depois da apresentação de vídeo educacional sobre o glaucoma, em Fortaleza (CE), que decidiram participar espontaneamente de uma campanha de prevenção ao glaucoma.

Resultados: Foram coletados 153 questionários, dentre os quais 130 foram considerados elegíveis, incluindo 65 antes da realização de vídeo educativo e 65 após. Antes e após o vídeo educativo, 80\% e $7 \%$, respectivamente, acreditavam que glaucoma não era mais comum em afro-americanos. As questões abordadas eram semelhantes a outras consolidadas na literatura, com o intuito de avaliar o grau de conhecimento dos participantes.

Conclusão: Foi possível observar um aumento no número de acertos após apresentação de material educativo.

\section{ABSTRACT}

Objective: To evaluate the population awareness of glaucoma.

Methods: A cross-sectional, descriptive, non-controlled study, using a questionnaire to collect data. The questions addressed sociodemographic profile and glaucoma, and were asked to individuals, before and after the presentation of an educational video on glaucoma. It was conducted in the city of Fortaleza (CE) and the respondents spontaneously decided to participate in a glaucoma prevention campaign.

Results: A total of 153 questionnaires were collected; in that, 130 were considered eligible, including 65 answered before and 65 after the presentation of the educational video. Before and after the educational video, $80 \%$ and $7 \%$, respectively, believed glaucoma was not more common in African Americans. The questions addressed were similar to others already consolidated in the literature, aiming to assess level of knowledge of participants.

Conclusion: An increase in number of correct answers was observed after presentation of the educational material. 


\section{INTRODUÇÃO}

O glaucoma é um grupo de doenças que causam danos estruturais ao nervo óptico e disfunção do campo visual. Na maior parte, ocorre de forma crônica, assintomática e, se não controlada, evolui progressivamente para cegueira. O glaucoma primário de ângulo aberto é o tipo predominante, sendo a principal causa de cegueira irreversível no mundo. ${ }^{(1)}$ Muitas pessoas só percebem que estão com problemas quando a doença progride significativamente, até afetar a visão central, já no final do processo patológico. ${ }^{(2-5)}$

Vários estudos buscam entender quais fatores estão associados à má aderência ao tratamento do glaucoma. ${ }^{(6)}$ Esses fatores não dependem de idade, sexo, nível de escolaridade, acuidade visual e capacidade de leitura. Entretanto, há associação com efeitos colaterais das medicações, má relação médico-paciente e desconhecimento sobre a doença. ${ }^{(7,8)}$

A apresentação tardia da doença está correlacionada com o baixo nível de conscientização sobre a doença, principalmente em países subdesenvolvidos. ${ }^{(7)}$ Assim, a conscientização acerca de sua natureza insidiosa e a importância da detecção e do tratamento precoces, na prevenção da cegueira, são fundamentais para o prognóstico dessa patologia. ${ }^{(7)}$

A importância de se investigar esse tema reside no fato de que a população brasileira, em sua maioria, tem acesso insuficiente a informações adequadas em relação à saúde em geral e sobre doenças crônicas, como o glaucoma. ${ }^{(7)}$ Acredita-se que esse desconhecimento leve ao diagnóstico tardio, à baixa adesão ao tratamento e, consequentemente, ao pior prognóstico visual.(9) Assim, faz-se necessário realizar campanhas para conscientizar e ensinar a população a respeito do glaucoma.

Nesse contexto, o presente estudo objetivou avaliar o conhecimento da população referente ao glaucoma, antes e depois de realizar uma atividade de conscientização sobre a doença.

\section{MÉTODOS}

Foi realizada uma pesquisa do tipo transversal descritiva, não controlada, com indivíduos que participaram espontaneamente de uma campanha de prevenção à cegueira pelo glaucoma em Fortaleza, capital do estado do Ceará, promovida pela Sociedade Cearense de Oftalmologia (SCO) e com o apoio dos principais shoppings da cidade. Essa campanha foi divulgada, previamente, por diferentes meios de comunicação do estado e ocorreu no período de 16 de maio de 2019 até o dia 26 de maio de 2019, correspondendo ao dia mundial de combate a essa doença. Esta pesquisa foi aprovada pelo Comitê de Ética em Pesquisa, com parecer 3.542.845 e CAAE: 18432619.0.0000.5049.

A amostra investigada foi escolhida por conveniência, tendo em vista que os entrevistadores abordavam as pessoas nos locais da campanha e perguntavam se elas gostariam de participar da pesquisa. O estudo continha voluntários de ambos os sexos, concordantes com o Termo de Conhecimento Livre e Esclarecido (TCLE). Inicialmente, o participante respondia ao questionário e, em seguida, assistia a um vídeo educativo sobre glaucoma, produzido pela SCO, com cerca de 15 minutos de duração. Ressalta-se o fato de que os autores da pesquisa possuíam autorização para a exibição do vídeo, via SCO conveniada ao local de exibição. Ao final do vídeo educativo, os participantes respondiam, novamente, as perguntas sobre a doença, para que fosse avaliada a mudança do conhecimento acerca da doença em questão.

Foram excluídas as pessoas que retornaram os questionários incompletos.

O instrumento de coleta de dados foi um questionário contendo perguntas sobre o perfil sociodemográfico do participante (como idade, sexo e nível de escolaridade) e seu conhecimento prévio acerca do glaucoma. Como base, foi utilizado um questionário previamente validado no idioma inglês, o Eye-Q Score(4). O questionário contou com dez perguntas que abordavam aspectos gerais da doença e consistiam de questões de resposta verdadeira ou falsa. Os questionários foram aplicados aos participantes por meio da plataforma on-line Microsoft Forms.

Os dados obtidos nos questionários aplicados foram dispostos no Microsoft Excel 2011 e analisados por meio do software Statistical Package for Social Sciences (SPSS), versão 23.o. O teste de Wilcoxon pareado foi aplicado para comparar os resultados do questionário sobre glaucoma antes e depois do vídeo educativo (software Prism 5.0 e GraphPad Instat, GraphPad Software Inc., CA, Estados Unidos). Valores de $\mathrm{p}<\mathrm{0}, 05$ foram considerados como estatisticamente significativos.

\section{RESULTADOS}

O estudo contou com amostra de 153 formulários preenchidos por voluntários em shoppings da cidade de Fortaleza. Dentre eles, 23 questionários foram excluídos da amostra, pois estavam preenchidos de forma incompleta, prejudicando a análise dos dados. Assim, restaram 130 formulários, os quais foram considerados elegíveis para análise dos dados, sendo que 65 foram obtidos antes da realização do vídeo educativo sobre as características do glaucoma e 65 foram obtidos dos mesmos voluntários após a transmissão do mesmo vídeo educativo. 
Quanto ao perfil dos voluntários, 56,92\% eram mulheres. A média de idade do estudo foi de 38,29 anos. Em relação à escolaridade, 26,15\% possuíam ensino médio completo, 63,07\% possuíam ensino superior completo, e 7,69\% possuíam apenas ensino fundamental incompleto. Com relação à doença, 69,23\% afirmavam não ter glaucoma com base em exames de rastreio prévios realizados em centros de acompanhamento oftalmológico, 20\% afirmavam não saber se possuíam, e apenas 7,69\% afirmaram possuir.

Com relação ao questionário de conhecimento acerca do glaucoma (Tabela 1), os indivíduos entrevistados, antes de assistirem ao vídeo educativo, em sua maioria (80\%) não reconhecia a etnia afro-brasileira como mais prevalente para o glaucoma; sabiam (82\%) da importância do fator hereditário no surgimento da doença; reconheciam (95\%) o caráter assintomático da doença e concordaram (75\%) com a maior probabilidade da doença acima dos 60 anos de idade. Quando questionados se a dor ocular era um sintoma frequente, $57 \%$ responderam que sim. No item sobre a existência de tratamento, 90\% responderam que era verdadeiro que a doença tem tratamento. Sobre a origem da doença relacionada ao aumento da pressão intraocular (PIO), cerca de 95\% responderam verdadeiro, sendo que $70 \%$ afirmavam que a visão não poderia ser restaurada, 91\% afirmavam ser falso que o único exame seria a medição da PIO, e 52\% afirmavam ser necessária a dilatação das pupilas para adequado exame do glaucoma.

Tabela 1. Resultado do Eye-Q Score

\begin{tabular}{|c|c|c|}
\hline & $\begin{array}{l}\text { Pré-teste } \\
\text { n (\%) }\end{array}$ & $\begin{array}{l}\text { Pós-teste } \\
\text { n (\%) }\end{array}$ \\
\hline $\begin{array}{l}\text { 1. O glaucoma é mais comum em } \\
\text { afro-americanos do que nos brancos? }\end{array}$ & $\begin{array}{c}\text { Falso }=45(80 \%) \\
\text { Verdadeiro }=11(20 \%)\end{array}$ & $\begin{array}{c}\text { Falso }=4(7 \%) \\
\text { Verdadeiro }=52(93 \%)\end{array}$ \\
\hline $\begin{array}{l}\text { 2. Pessoas que têm parentes } \\
\text { próximos com glaucoma estão em } \\
\text { maior risco? }\end{array}$ & $\begin{array}{c}\text { Falso }=10(18 \%) \\
\text { Verdadeiro }=46(82 \%)\end{array}$ & $\begin{aligned} \text { Falso } & =2(3 \%) \\
\text { Verdadeiro } & =54(97 \%)\end{aligned}$ \\
\hline $\begin{array}{l}\text { 3. Uma pessoa pode ter glaucoma e } \\
\text { não saber? }\end{array}$ & $\begin{aligned} \text { Falso } & =3(5 \%) \\
\text { Verdadeiro } & =53(95 \%)\end{aligned}$ & $\begin{array}{c}\text { Falso }=0 \\
\text { Verdadeiro }=56(100 \%)\end{array}$ \\
\hline $\begin{array}{l}\text { 4. Pessoas com mais de } 60 \text { anos } \\
\text { têm mais probabilidade de contrair } \\
\text { glaucoma? }\end{array}$ & $\begin{array}{c}\text { Falso }=14(25 \%) \\
\text { Verdadeiro }=42(75 \%)\end{array}$ & $\begin{aligned} \text { Falso } & =5(9 \%) \\
\text { Verdadeiro } & =51(91 \%)\end{aligned}$ \\
\hline $\begin{array}{l}\text { 5. Dor nos olhos é frequentemente } \\
\text { um sintoma de glaucoma? }\end{array}$ & $\begin{array}{c}\text { Falso }=24(43 \%) \\
\text { Verdadeiro }=32(57 \%)\end{array}$ & $\begin{array}{c}\text { Falso }=34(60 \%) \\
\text { Verdadeiro }=22(40 \%)\end{array}$ \\
\hline 6. O glaucoma pode ser tratado? & $\begin{array}{c}\text { Falso }=6(10 \%) \\
\text { Verdadeiro }=50(90 \%)\end{array}$ & $\begin{array}{c}\text { Falso }=2(3 \%) \\
\text { Verdadeiro }=54(97 \%)\end{array}$ \\
\hline $\begin{array}{l}\text { 7. O glaucoma é causado pelo } \\
\text { aumento da pressão ocular? }\end{array}$ & $\begin{array}{c}\text { Falso }=3(5 \%) \\
\text { Verdadeiro }=53(95 \%)\end{array}$ & $\begin{array}{c}\text { Falso }=5(9 \%) \\
\text { Verdadeiro }=51(91 \%)\end{array}$ \\
\hline $\begin{array}{l}\text { 8. A visão perdida do glaucoma pode } \\
\text { ser restaurada? }\end{array}$ & $\begin{array}{c}\text { Falso }=39(70 \%) \\
\text { Verdadeiro }=17(30 \%)\end{array}$ & $\begin{array}{c}\text { Falso }=46(82 \%) \\
\text { Verdadeiro }=10(18 \%)\end{array}$ \\
\hline $\begin{array}{l}\text { 9. Um exame completo de glaucoma } \\
\text { consiste apenas em medir a pressão } \\
\text { ocular? }\end{array}$ & $\begin{array}{c}\text { Falso }=51(91 \%) \\
\text { Verdadeiro }=5(9 \%)\end{array}$ & $\begin{array}{l}\text { Falso }=52(93 \%) \\
\text { Verdadeiro }=4(7 \%)\end{array}$ \\
\hline $\begin{array}{l}\text { 10. Pessoas em risco de glaucoma } \\
\text { devem ter um as pupilas dilatadas } \\
\text { durante o exame oftalmológico? }\end{array}$ & $\begin{array}{c}\text { Falso }=27(48 \%) \\
\text { Verdadeiro }=29(52 \%)\end{array}$ & $\begin{array}{c}\text { Falso }=24(43 \%) \\
\text { Verdadeiro }=32(57 \%)\end{array}$ \\
\hline
\end{tabular}

Ao final da exibição do vídeo educativo, a maioria dos indivíduos melhora seus índices, reconhecendo aspectos relevantes do glaucoma, com significância estatística ( $\mathrm{p}=\mathrm{0}, 0308)$, considerando como nota dez quem acertou todos as perguntas e zero para quem errou todas (cada questão valendo um ponto); 75,38\% melhoraram o número de acertos, 20\% mantiveram e 4,61\% pioraram (Figuras 1 a 3).

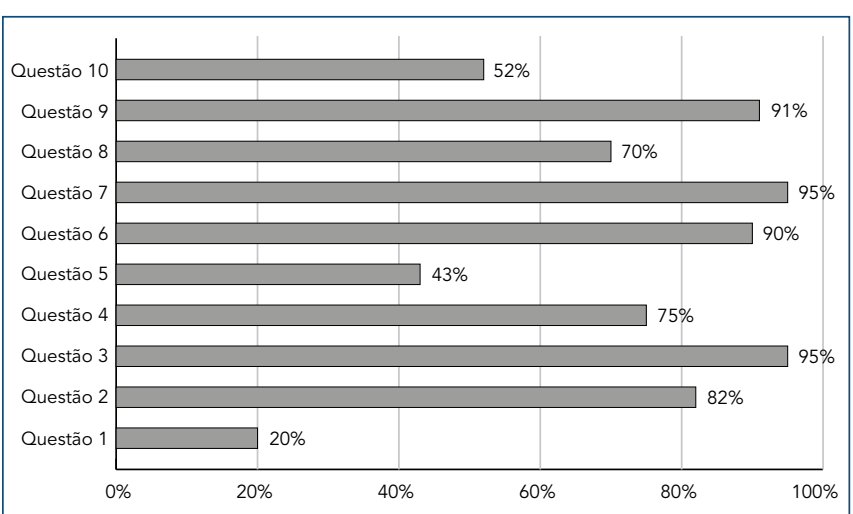

Figura 1. Percentual de acertos de cada questão pré-vídeo.

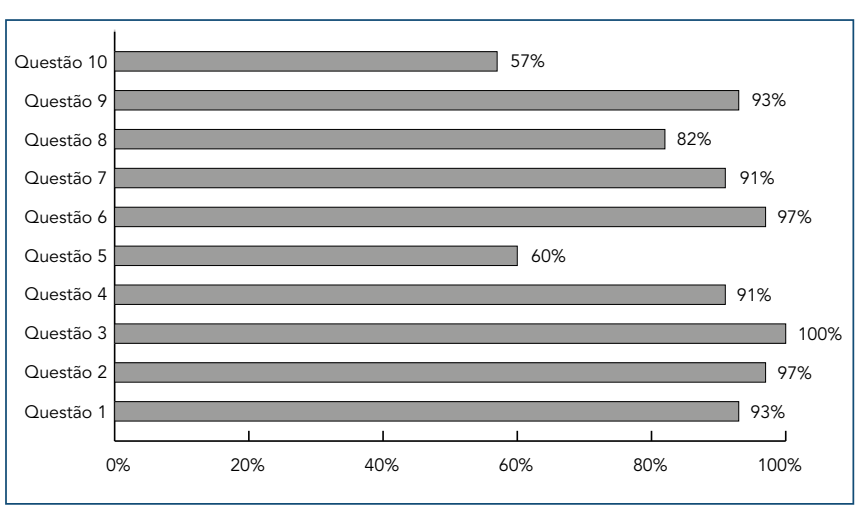

Figura 2. Percentual de acertos de cada questão pós-vídeo.

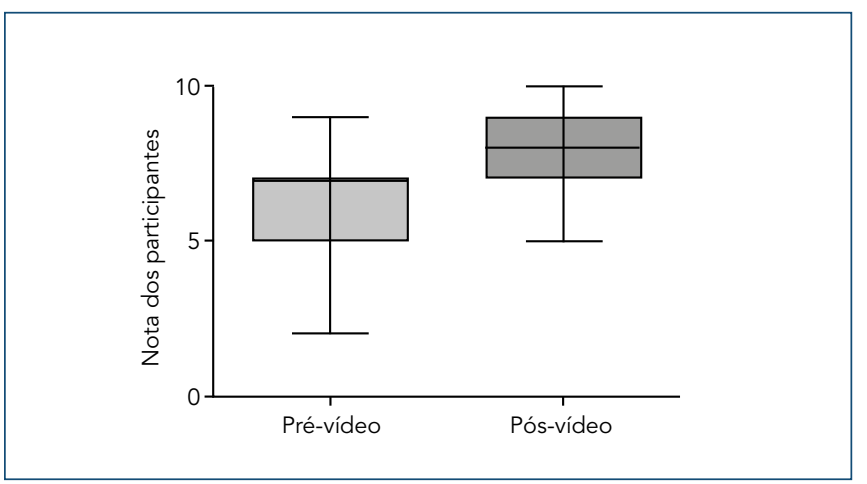

Dados apresentados na forma de percentis 25 e 75 (linhas inferiores e superiores, respectivamente) e traços inferiores e superiores, indicando os limites observados. Valor de $p<0,05$ foi considerado como estatisticamente significativo.

Figura 3. Comparação entre as respostas ao questionário acerca do glaucoma, antes $(n=65)$ e após $(n=65)$ os entrevistados terem assistido ao vídeo educativo sobre essa patologia.

\section{DISCUSSÃO}

O Eye-Q Score foi desenvolvido como uma ferramenta de saúde pública para avaliar o conhecimento em pacientes 
com glaucoma inicial, suspeita de glaucoma ou fatores de risco para o glaucoma. ${ }^{(4)} \mathrm{A}$ fim de melhor avaliar o conhecimento do paciente de glaucoma e monitorar a aprendizagem durante o atendimento clínico de rotina, uma avaliação padronizada do conhecimento sobre o glaucoma seria fundamental. Isso seria especialmente útil à luz da literatura que defende que a adesão em pacientes com glaucoma é frequentemente ruim e tende a melhorar com um melhor conhecimento acerca da doença. ${ }^{(4)}$

A prevalência de glaucoma é de 2,93\% entre os $40 \mathrm{e}$ 80 anos de idade, aumentando para 10\% nas pessoas acima de 90 anos. A média de idade encontrada, em alguns estudos, é de 63,5 anos. ${ }^{\left({ }^{9}\right)} \mathrm{Um}$ fato que pode ter corroborado para que a média de idade deste estudo tenha sido menor do que o encontrado na literatura para o glaucoma foi o local de realização da pesquisa, pois, em shopping, comumente se encontram adultos jovens. No entanto, esta porcentagem encontrada de 38,29 anos torna-se relevante, uma vez que aborda a população antes do acometimento da doença, sendo o público-alvo da SCO, visando à prevenção da patologia nessa região. Ademais, é importante ressaltar que a campanha foi composta por exposição a vídeo educativo sobre o glaucoma e aplicação do questionário de conhecimento da população acerca dessa doença, não havendo, portanto, realização de exames médicos. Também vale destacar que, apesar de a amostra ser pequena, foi possível entrevistar pessoas de diversas escolaridades, idades e de ambos os sexos.

Em relação às perguntas presentes no questionário, a afirmativa de que o glaucoma é mais comum em afro-americanos do que em brancos é verdadeira, pois, em estudo financiado pelo National Eye Institute (NEI), pesquisadores da John Hopkins University evidenciaram que o glaucoma é três a quatro vezes mais provável nesse grupo populacional, além de ser seis vezes mais provável de causar cegueira nesses indivíduos, tendo em vista uma maior gravidade da doença nessa etnia. ${ }^{(8-10)} \mathrm{O}$ presente estudo reaplicou o questionário após os participantes assistirem ao vídeo educativo, demonstrando que cerca de $23 \%$ das pessoas mudaram de opinião.

Em relação à história familiar como fator de risco, apesar de não ter sido estabelecida nenhuma base hereditária, sabe-se que é mais provável de ter glaucoma caso algum membro da família com parentesco em primeiro grau tenha a doença. ${ }^{\left({ }^{8,9}\right)}$ Quando se analisam as respostas obtidas, percebe-se um aumento de $10 \%$ no acerto da pergunta após assistir ao vídeo.

Em relação às respostas das pessoas sobre o aspecto assintomático da doença, 95\% reconheceram-na como assintomática, contudo, os 5\% restante mudaram de opinião ao assistir o vídeo. Com relação ao sintoma dor no glaucoma, visto que geralmente pessoas com glaucoma não sentem dores, ${ }^{(8,9)}$ no presente estudo $42 \%$ tinham assinalado ausência de dor, mas, após o vídeo educativo, esse número aumentou apenas para 60\%, demonstrando que a população tem muito arraigado esse conceito de dor no processo de glaucoma.

Estudos populacionais demonstram que a população com mais de 60 anos possui maior risco de desenvolver o glaucoma, ${ }^{(8-10)}$ fato este aprendido por $16 \%$ das pessoas que assistiram ao vídeo.

Segundo a literatura mundial, o glaucoma pode ser tratado com terapia medicamentosa, cirurgia incisional ou a laser. ${ }^{(8,11)}$ A maioria dos entrevistados demonstrou $(90 \%)$ conhecimento acerca do tratamento, aumentando em $6 \%$ esse conhecimento após o vídeo educativo.

A pergunta sobre a causa do glaucoma ser o aumento da PIO tem como resposta falsa, já que a elevação da PIO significa que a pessoa está em risco aumentado para desenvolver glaucoma, mas não indica que a pessoa tenha a doença. ${ }^{(7,10,12)}$ A presença do glaucoma ocorre quando há lesão do nervo óptico e esta pode ocorrer mesmo na vigência de PIO normal. ${ }^{(8,13,14)}$ Antes do vídeo, somente uma pequena parcela de participantes $(6 \%)$ concordara que essa pergunta era falsa, e, após o vídeo, esse valor elevou-se somente para 9\%, demonstrando o peso da PIO elevada como causa ou conceito da doença na crença da população local.

A pergunta a respeito da possibilidade de restaurar a visão perdida é falsa, visto que o comprometimento visual é irreversível. No entanto, a detecção precoce e o tratamento podem impedir a perda da visão. ${ }^{(8-10,13)}$ Em relação às respostas antes e após o vídeo, houve uma melhora, pois, no início, 72\% afirmavam ser falso, e, após assistir ao vídeo, cerca de $8 \%$ das pessoas mudaram de opinião e falaram que essa afirmativa era falsa.

A pergunta a respeito do exame para o rastreio do glaucoma aborda conhecimentos de PIO aferida pela tonometria. Embora seja uma parte importante do exame oftalmológico, ele não é suficiente para detectar o glaucoma. ${ }^{(8,11,13,15)}$ Deve-se avaliar como está o nervo óptico, por meio da dilatação das pupilas, além de realizar a análise do campo visual. ${ }^{(8,11,13,15,16)}$ De acordo com a resposta da população avaliada, a maioria (91\%) avaliou que são necessários outros exames, além da tonometria, para o correto diagnóstico dessa doença, no entanto, isso contradiz a taxa elevada (94\%) de pessoas que afirmaram ser a PIO elevada o conceito de glaucoma. 
A respeito da importância de dilatar as pupilas para a realização do exame em pessoas em risco de glaucoma, essa pergunta é verdadeira, pois o exame oftalmológico, por meio da midríase, é a melhor maneira de diagnosticar a neuropatia glaucomatosa, devendo ser realizado anualmente. ${ }^{\left({ }^{8}\right)}$ Antes de assistir ao vídeo, 52\% afirmavam que era preciso dilatar as pupilas; ao fim, 58\% afirmavam que essa pergunta essa verdadeira.

Neste estudo, a pontuação média pré-teste foi de 6,27 e o desvio-padrão, de 1,57; no pós-teste, a média foi de 7,8, e o desvio-padrão foi de 1,26. Esse dado demonstra que o vídeo educativo contribuiu para a aprendizagem dos participantes. Rao et al. aplicaram esse mesmo questionário para avaliar o conhecimento sobre glaucoma em população de pacientes que possuía essa doença e encontraram nota média de 7,3. ${ }^{(4)}$

O presente estudo apresentou limitações. Dentre as quais, pode-se destacar a não validação do questionário Eye-Q Score para a língua portuguesa, o pequeno tamanho da amostra (tendo em vista o tamanho da população da capital do Ceará) e o viés de seleção, considerando a amostra uma maior quantidade de indivíduos adultos jovens, impossibilitando uma melhor comparação com os dados na literatura vigente.

\section{CONCLUSÃO}

Houve aumento dos acertos ao se analisarem as respostas de antes e depois do vídeo educativo. O vídeo educacional, assim, contribuiu para o aumento do conhecimento da população avaliada, demonstrando que esse tipo de tecnologia sobre o glaucoma é uma boa medida para promover educação em saúde da população em geral.

\section{AGRADECIMENTOS}

À Sociedade Cearense de Oftalmologia, pelo convite para a realização da campanha para conscientização sobre glaucoma. Sua ajuda foi de crucial importância para a realização deste estudo, bem como para informar parte da população sobre este assunto de grande relevância.

\section{REFERÊNCIAS}

1. Quigley HA, Broman AT. The number of people with glaucoma worldwide in 2010 and 2020. Br J Ophthalmol. 2006;90(3):262-7.

2. Couto I, Marins D, Santo F, Neves P. Knowledge and practice: education in health as link facilitative in the care process. Rev Pesqui Cuidado Fundament. 2012;5(1):3485-92

3. Rosdahl JA, Muir KW. Finding the best glaucoma questionnaire: a qualitative and quantitative evaluation of glaucoma knowledge assessments. Clin Ophthalmol. 2015;9:1845-52.

4. Rao V, Peralta E, Rosdahl J. Validation of a glaucoma knowledge assessment in glaucoma patients. Clin Ophthalmol. 2016;10:1913-8.

5. Kizor-Akaraiwe NN, Monye HI, Okeke S. Awareness and knowledge about glaucoma and proportion of people with glaucoma in an urban outreach programme in Southeast Nigeria. BMJ Open Ophthalmol. 2017;1(1):e000018.

6. Castro NA, Mesquita WA. Não-adesão à terapêutica medicamentosa do glaucoma. Arq Bras Oftalmol. 2008;71(2):207-14.

7. Costa VP, Vasconcelos JP, Pelegrino M, Kara-José N. O que os pacientes sabem sobre glaucoma? Arq Bras Oftalmol. 1995;58(1):36-41.

8. Prevent Blindness America (PBA) Eye Q Test. [cited 2021 Jun 1]. Available from: http://preventblindness.org/wp-content/uploads/2011/06/MK19_ GlaucEyeQ_2017.pdf

9. Loureiro FL, Felix KA. Perfil clínico e epidemiológico dos pacientes com glaucoma atendidos em um ambulatório no interior da Amazônia. Rev Bras Oftalmol. 2020;79(1):12-20.

10. Gonçalves MR, Guedes MM, Chaves MA, Pereira CC, Otton R. Análise dos fatores de risco e epidemiologia em campanha de prevenção da cegueira pelo glaucoma em João Pessoa, Paraíba. Rev Bras Oftalmol. 2013;72(6):396-9

11. Vieira AA, Guedes RA, Vieira RC, Guedes VM. Percepção do paciente portador de glaucoma e os diferentes tipos de tratamento (clínico versus cirúrgico). Rev Bras Oftalmol. 2015; 74(4):235-40.

12. Silva MJ, Temporini ER, Neustein I, Araújo ME. Conhecimento sobre prevenção e tratamento de glaucoma entre pacientes de unidade hospitalar. Arq Bras Oftal. 2004;67(5):785-90.

13. Luu KT, Raber SR, Nickens DJ, Vicini P. A model-based meta-analysis of the effect of latanoprost chronotherapy on the circadian intraocular pressure of patients with glaucoma or ocular hypertension. Clin Pharmacol Ther. 2010;87(4):421-5

14. Esporcatte BL, Tavares IM. Normal-tension glaucoma: an update. Arq Bras Oftalmol. 2016;79(4):270-6

15. Martins S, Mendes M, Guedes R. Nível de conhecimento sobre glaucoma primário de ângulo aberto entre os estudantes de medicina. Rev Bras Oftalmol. 2014;73(5):302-7

16. Araújo JD, De Paiva AC, De Almeida JD, Silva Neto OP, De Sousa JA, Silva $A C$, et al. Diagnóstico de glaucoma em imagens de fundo de olho utilizando os índices de diversidade de Shannon e Mclntosh. In: Simpósio Brasileiro de Computação Aplicada à Saúde (SBCAS), 17, 2017, São Paulo. Anais. Porto Alegre: Sociedade Brasileira de Computação, 2017. DOI: https://doi.org/10.5753/sbcas.2017.3698 